\title{
The Contribution of Thermally Scattered Electrons to Atomic Resolution Elemental Maps
}

R.E.A. Williams ${ }^{1}$, B.D. Forbes ${ }^{2}$, A.J. D’Alfonso², R. Srinivasan', B. Freitag ${ }^{3}$, D.O. Klenov ${ }^{3}$, H.L. Fraser $^{1}$, L.J. Allen ${ }^{2}$ and D.W. McComb ${ }^{1}$

${ }^{1}$ Department of Materials Science and Engineering, The Ohio State University, 477 Watts Hall, 2041 College Road, Columbus, OH 43210, USA

${ }^{2}$ School of Physics, University of Melbourne, Parkville, Victoria 3010, Australia

${ }^{3}$ FEI Company, Building AAE, Achtseweg Noord 5, Eindhoven, The Netherlands (Dated: May 10, 2012)

The recent technological improvements in microscope electronics and stability have afforded researchers the ability to collect atomic resolution elemental maps of various materials using electron energy-loss spectroscopy (EELS) and energy dispersive x-ray (EDX) analysis in a scanning transmission electron microscope (STEM). The inelastic scattering signal, for EELS atomic resolution maps, is collected post specimen and is composed of electrons that have lost energy on passing through the specimen, while the collection aperture defines/limits the angular collection range. Compositional maps of salient species may then be processed from a subset of electrons falling into a suitable energy loss window above the threshold energy for a given core-loss edge. Moreover, it is often impossible to collect all pertinent spectral data simultaneously, introducing experimental uncertainty in collection.

Conversely, during collection of x-rays with modern quad-detector silicon drift detectors $(\sim 1 \mathrm{sr})$ for EDX spectral mapping, all possible kinematics of the inelastically scattered fast electron are effectively sampled, additionally no restriction to scattering in the forward direction is imposed by the EELS spectrometer. Furthermore, the energy window is effectively all possible energies above the threshold. It is expected that EELS spectral mapping, with a large detector aperture and a large energy window, would have similar underlying physics to EDX-based chemical mapping.

For this work we present atomic resolution EELS and EDX maps of the oxygen K-shell in $<001<$ strontium titanate. Considering previously reported EELS elemental maps, it was observed that thermal diffuse scattering can reduce the signal from an on-column relative to off-column probe position by depleting the elastically scattered probe on the column through thermal diffuse scattering (TDS).[1,2] Therefore, it was expected that TDS would redistribute the electron flux away from the $\mathrm{Ti} / \mathrm{O}$ atomic column, effectively reducing signal strength for both the EELS and EDX cases resulting in a lower signal when the probe is on the Ti/O columns than when it is on the $\mathrm{O}$ columns. However, the results, shown in Figure 1, appeared anomalous since substantially more signal is obtained when the STEM probe is above the columns containing both Ti/O than those containing only $\mathrm{O}$.

Along the $\langle 001<$ orientation, based upon stoichiometry, the density of oxygen in both $\mathrm{Ti} / \mathrm{O}$ and $\mathrm{O}$ columns is identical. Using theory, it was shown that understanding the direct contribution from thermally scattered electrons is critical to interpreting atomic resolution EELS and EDS results correctly. These results were compared to EELS and EDS elemental maps of $\langle 110<$ strontium titanate. The experimental results presented are not directly interpretable, however, they are in agreement with simulations from first-principles such as those presented.[3] 


\section{References}

[1] M. Bosman, et al, Physical Review Letters 99, 086102 (2007)

[2] P. Wang, et al, Physical Review Letters 101, 236102 (2008).

[3] B.D. Forbes, et al, Phys. Rev. B 86, 024108 (2012)

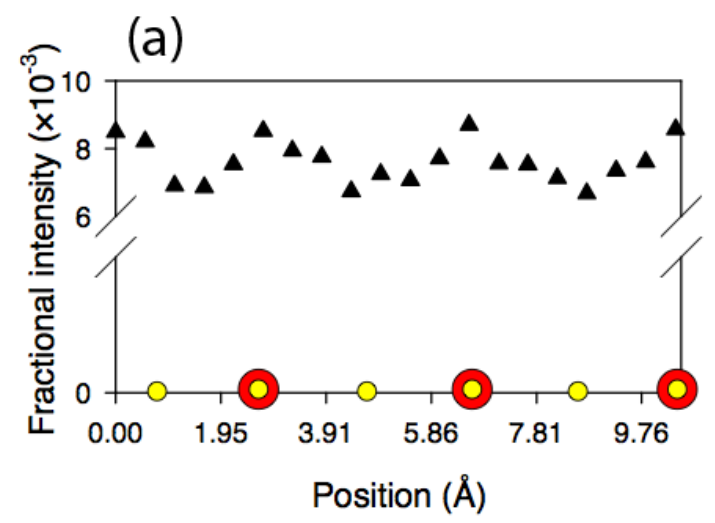

(b)

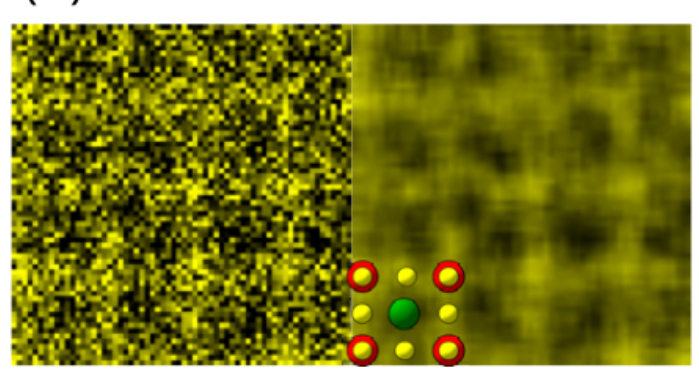

Figure 1 (a): An EELS line scan from $\langle 001<$ strontium titanate monitoring the oxygen Kshell signal along successive $\mathrm{Ti} / \mathrm{O}$ and pure $\mathrm{O}$ columns. The positions of the $\mathrm{Ti} / \mathrm{O}$ (red and smaller inset yellow circles) and $\mathrm{O}$ columns (small yellow circles) are indicated. (b) An EDX elemental map of $\langle 001<$ strontium titanate monitoring the oxygen $\mathrm{K} \alpha$ line for a single scan (raw data) and with averaging. 